\title{
PERFECT IMAGES OF ČECH-ANALYTIC SPACES
}

\author{
R. W. HANSELL AND SHIHO PAN
}

(Communicated by Franklin D. Tall)

\begin{abstract}
A completely regular Hausdorff space $X$ is Čech-analytic if $X$ is the result of the Souslin operation applied to the locally compact sets in some (equivalently, any) compactification. We prove that Čech-analytic spaces are preserved under general perfect maps, thus settling a question raised by the late Z. Frolík.
\end{abstract}

\section{INTRODUCTION}

In 1980 Fremlin [2] introduced the class of Čech-analytic spaces as a common generalization of the classes of $K$-analytic spaces and nonseparable analytic metric spaces "in much the same way that the Čech-complete spaces form a common generalization of the locally compact Hausdorff spaces and the complete metric spaces". In [2] many of the standard stability properties associated with a class of "analytic spaces" were established. In particular, the family of Čech-analytic spaces was shown to be closed to the Souslin operation (hence, to countable intersections and unions), projections parallel to the space $\omega^{\omega}$ of irrational numbers, and countable products.

Perhaps the most important stability property of separable analytic metric spaces and $K$-analytic spaces is that these are preserved by arbitrary continuous maps. Of course, this fails in the nonseparable case since any space is a continuous image of a discrete metric space, and something more than continuity is required; see [6]. However, all previous classes of analytic spaces, of the separable or nonseparable type, are known to be invariant under perfect maps (i.e., continuous closed maps with compact fibers).

Soon after Fremlin introduced the class of Čech-analytic spaces, Frolik [4] recognized the importance of determining whether or not Čech-analytic spaces are preserved by perfect maps. In 1989 Frolík [5] gave an affirmative answer in the case when the image space is hereditarily paracompact (hence when metrizable), extending an earlier result of Fremlin [3] when the image space is hereditarily Lindelöf. The purpose of the present paper is to show that Čech-analytic spaces are preserved under perfect maps in general.

The paper concludes with a brief section on bi-Čech-analytic spaces, that is,

Received by the editors December 19, 1992 and, in revised form, April 12, 1993.

1991 Mathematics Subject Classification. Primary 54H05, 54C10.

Key words and phrases. Čech-analytic space, perfect map, Souslin- $(\mathscr{F} \wedge \mathscr{G})$ set. 
spaces $X$ for which there is some compactification $X^{*}$ such that both $X$ and $X^{*} \backslash X$ are Čech-analytic spaces. As an application of our main result we show that this concept is absolute in that $X$ and $X^{*} \backslash X$ are Čech-analytic spaces for any compactification $X^{*}$.

\section{MAIN THEOREM}

Recall that if $\mathscr{E}$ is any collection of sets, by a Souslin-( $\mathscr{E})$ set we mean a set of the form

$$
S=\bigcup_{s \in \omega^{\omega}} \bigcap_{n \in \omega} E_{s \mid n}
$$

corresponding to some (countable) collection $\left\{E_{s \mid n}: s \mid n \in \omega^{n}, n \in \omega\right\} \subset \mathscr{E}$, where $s \mid n=s_{1}, s_{2}, \ldots, s_{n}$ and refers to the first $n$ terms of the sequence $s \in \omega^{\omega}$. The set $S$ is also said to be the result of the Souslin operation applied to $\mathscr{E}$. It is well known that a completely regular Hausdorff space $X$ is $K$-analytic if and only if it is a Souslin- $\mathscr{F}$ set in some (equivalently, any) compactification and that such spaces are necessarily Lindelöf. As is customary, we use $\mathscr{F}$ to denote the family of closed sets and $\mathscr{G}$ the family of open sets of a given space. By a $\mathscr{F} \wedge \mathscr{G}$ set we mean one that is the intersection of a closed set with an open set, and $\mathscr{F} \cup \mathscr{G}$ refers to the usual set-theoretic union of the families $\mathscr{F}$ and $\mathscr{G}$. Note that if $\mathscr{B}$ denotes the family of Borel sets for a space $X$, then each of the families Souslin- $(\mathscr{F} \cup \mathscr{G})$, Souslin- $(\mathscr{F} \wedge \mathscr{G})$, and Souslin- $(\mathscr{B})$ coincide, since each Borel set is Souslin- $(\mathscr{F} \cup \mathscr{G})$ and the class Souslin- $(\mathscr{B})$ is invariant under the Souslin operation.

A completely regular Hausdorff space $X$ is Čech-analytic if it is a Souslin$(\mathscr{F} \wedge \mathscr{G})$ set in some compactification. Fremlin [2] has shown that a Cechanalytic space will be a Souslin-(Borel) set in any completely regular Hausdorff embedding, and so these are the "absolute Souslin-(Borel) sets" relative to the class of completely regular Hausdorff spaces.

A key ingredient of the proof of our main theorem is the following property of compact sets.

Lemma 2.1. Let $\mathscr{U}$ be an open cover of a nonempty compact set $K$ in some topological space $X$. Then there is $\mathscr{W} \subset \mathscr{U}$ such that $K^{*} \equiv K \backslash \bigcup \mathscr{W}$ is nonempty and, whenever $K^{*} \cap U \neq \varnothing$ for some $U \in \mathscr{U}, K^{*} \subset U$.

Proof. Let $\Omega$ denote the set of all $\mathscr{W} \subset \mathscr{U}$ such that $K \backslash \bigcup \mathscr{W}$ is nonempty, and note that $\varnothing \in \Omega$. Partially order $\Omega$ by set inclusion. Let $\Gamma$ be any chain in $\Omega$, and let $\mathscr{V}$ denote the union of all members of $\Gamma$. Suppose, if possible, that $K \backslash \cup \mathscr{V}$ is empty. By the compactness of $K$ we can find a finite sequence $\mathscr{V}_{1} \subset \cdots \subset \mathscr{V}_{n}$ in $\Gamma$ such that the union of the $\mathscr{V}_{k}$ and hence also $\mathscr{V}_{n}$ is a cover of $K$. But this contradicts the fact that $\mathscr{V}_{n}$ belongs to $\Omega$. It follows by Zorn's Lemma that $\Omega$ has a maximal element.

Let $\mathscr{W}$ be such a maximal element, and let $K^{*}=K \backslash \bigcup \mathscr{W}$. Suppose $K^{*} \cap$ $U \neq \varnothing$, for some $U \in \mathscr{U}$, and that $K^{*} \not \subset U$. But then $\mathscr{W} \cup\{U\}$ belongs to $\Omega$, so $U \in \mathscr{W}$ by the maximality of $\mathscr{W}$. But this contradicts the fact that $K^{*} \cap U \neq \varnothing$. Hence we must have $K^{*} \subset U$ as required.

Theorem 2.2. Let $X$ be a Čech-analytic space, and suppose $f: X \rightarrow Y$ is a perfect continuous onto map. If $Y$ is completely regular Hausdorff, then $Y$ is Cech-analytic. 
Proof. Let $\beta X$ be the Stone-Čech compactification of $X$, and let $Y^{*}$ be some compactification of $Y$. Since $X$ is a Souslin- $(\mathscr{F} \wedge \mathscr{G})$ set in $\beta X$, we have

$$
X=\bigcup_{s \in \omega^{\omega}} \bigcap_{n \in \omega} F_{s \mid n} \cap G_{s \mid n},
$$

where $F_{s \mid n}$ is closed and $G_{s \mid n}$ is open in $\beta X$ for each $s \in \omega^{\omega}$. Let $\Delta$ denote all nonempty finite subsets of $\bigcup_{n \in \omega} \omega^{n}$, and let

$$
\Gamma=(\Delta \times \omega)^{\omega} \text {. }
$$

Our objective is to define closed sets $F_{\gamma \mid n}^{*}$ and open sets $G_{\gamma \mid n}^{*}$ in $Y^{*}$, for each $\gamma \mid n \in(\Delta \times \omega)^{n}$ and $n \in \omega$, such that

$$
Y=\bigcup_{\gamma \in \Gamma} \bigcap_{n \in \omega} F_{\gamma \mid n}^{*} \cap G_{\gamma \mid n}^{*}
$$

As $\Delta \times \omega$ is countable, it will then follow that $Y$ is Čech-analytic.

Given $n \in \omega$ and $\gamma \mid n \in(\Delta \times \omega)^{n}$, suppose

$$
\gamma \mid n=\left(R_{1}, s_{1}\right), \ldots,\left(R_{n}, s_{n}\right) .
$$

For each $R_{k}(k=1, \ldots, n)$ let

$$
G\left(R_{k}\right)=\bigcup\left\{G_{\xi}: \xi \in R_{k}\right\},
$$

where $G_{\xi}$ corresponds to the open set in the representation (1). Now define

$$
\begin{aligned}
& G_{\gamma \mid n}=G\left(R_{1}\right) \cup \cdots \cup G\left(R_{n}\right), \\
& G_{\gamma \mid n}^{*}=Y^{*} \backslash f^{\beta X}\left[X^{*} \backslash\left(G_{\gamma \mid n} \cup G_{s \mid n}\right)\right], \\
& F_{\gamma \mid n}=\bigcap_{m=1}^{n} F_{s \mid m} \backslash G_{\gamma \mid n},
\end{aligned}
$$

and

$$
F_{\gamma \mid n}^{*}=f^{\beta X}\left[F_{\gamma \mid n}\right],
$$

where $f^{\beta X}$ is the Stone-Čech extension of $f$ to $\beta X$. Since $f^{\beta X}$ is a closed map from $\beta X$ onto $Y^{*}$, it follows that $G_{\gamma \mid n}^{*}$ is an open set and $F_{\gamma \mid n}^{*}$ is a closed set relative to $Y^{*}$.

Note that for any $\gamma=\left(R_{1}, s_{1}\right),\left(R_{2}, s_{2}\right), \ldots \in \Gamma$ we have $F_{\gamma \mid 1} \supset F_{\gamma \mid 2} \supset \ldots$ and that

$$
y \in G_{\gamma \mid n}^{*} \text { if and only if }\left(f^{\beta X}\right)^{-1}(y) \subset G_{\gamma \mid n} \cup G_{s \mid n} .
$$

Also note that $f^{-1}(y)=\left(f^{\beta X}\right)^{-1}(y)$ for any $y \in Y$, since for a perfect map $f$ we have $f^{\beta X}(\beta X \backslash X) \subset Y^{*} \backslash Y$ [1, p. 239].

Now suppose $y \in Y$ and let us find a $\gamma \in \Gamma$ such that $y \in \bigcap_{n \in \omega} F_{\gamma \mid n}^{*} \cap G_{\gamma \mid n}^{*}$. We first apply Lemma 2.1 to the nonempty compact set $f^{-1}(y)=\left(f^{\beta X}\right)^{-1}(y)$ and the open cover $\mathscr{U}=\left\{G_{s \mid n}: s \mid n \in \omega^{n}, n \in \omega\right\}$. Hence there is a nonempty compact set $K$ of the form $f^{-1}(y) \backslash \bigcup \mathscr{W}$, for some $\mathscr{W} \subset \mathscr{U}$, such that

$$
K \cap G_{s \mid n} \neq \varnothing \Rightarrow K \subset G_{s \mid n} \text {. }
$$

Fix some $x \in K$ and let $s$ be any element of $\omega^{\omega}$ such that $x \in \bigcap_{n \in \omega} F_{s \mid n} \cap G_{s \mid n}$. It follows from (2) that $f^{-1}(y)$ is covered by $\mathscr{W} \cup\left\{G_{s \mid n}\right\}$ for each $n \in \omega$, so there are finite subsets $R_{n} \subset \omega \cup \omega^{2} \cup \cdots$ such that

$$
f^{-1}(y) \subset G\left(R_{n}\right) \cup G_{s i n},
$$


where $G\left(R_{n}\right)=\bigcup\left\{G_{\xi}: \xi \in R_{n}\right\}$ and $G_{\xi} \in \mathscr{W}$ for each $\xi \in R_{n}$. Put

$$
\gamma=\left(R_{1}, s_{1}\right),\left(R_{2}, s_{2}\right), \ldots \in \Gamma \text {. }
$$

It follows immediately from (3) and our observation above that $y \in G_{y \mid n}^{*}$ for each $n \in \omega$. Also, since $x \in K \subset X^{*} \backslash \bigcup \mathscr{W}$ and $G\left(R_{n}\right) \subset \bigcup \mathscr{W}$ for each $n$, we have

$$
x \in \bigcap_{m=1}^{n} F_{s \mid m} \backslash\left(G\left(R_{1}\right) \cup \cdots \cup G\left(R_{n}\right)\right),
$$

hence $x \in F_{\gamma \mid n}$ and $y \in F_{\gamma \mid n}^{*}$. It follows that

$$
Y \subset \bigcup_{\gamma \in \Gamma} \bigcap_{n \in \omega} F_{\gamma \mid n}^{*} \cap G_{\gamma \mid n}^{*}
$$

Conversely, suppose $y \in \bigcap_{n \in \omega} F_{\gamma \mid n}^{*} \cap G_{\gamma \mid n}^{*}$ and that $\gamma=\left(R_{1}, s_{1}\right),\left(R_{2}, s_{2}\right), \ldots$ $\in \Gamma$. Since $y \in G_{\gamma \mid n}^{*}$, as noted above we have

$$
\left(f^{\beta X}\right)^{-1}(y) \subset G_{\gamma \mid n} \cup G_{s \mid n}
$$

for each $n$. Also, since $\left\{F_{\gamma \mid n}\right\}$ is a decreasing sequence of nonempty compact sets in $\beta X$, we have

$$
f^{\beta X}\left(\bigcap_{n \in \omega} F_{\gamma \mid n}\right)=\bigcap_{n \in \omega} f^{\beta X}\left(F_{\gamma \mid n}\right)=\bigcap_{n \in \omega} F_{\gamma \mid n}^{*} .
$$

Since $y \in \bigcap_{n \in \omega} F_{\gamma \mid n}^{*}$, there exists some $x \in \beta X$ such that

$$
x \in\left(f^{\beta X}\right)^{-1}(y) \cap \bigcap_{n \in \omega} F_{\gamma \mid n} \subset \bigcap_{n \in \omega}\left[\left(G_{\gamma \mid n} \cup G_{s \mid n}\right) \cap F_{\gamma \mid n}\right] .
$$

Hence, by the definitions of $F_{\gamma \mid n}$ and $G_{\gamma \mid n}$, for each $n \in \omega$ we have

$$
x \in\left(F_{s \mid n} \backslash G_{\gamma \mid n}\right) \cap\left(G_{\gamma \mid n} \cup G_{s \mid n}\right),
$$

so $x \in \bigcap_{n \in \omega} F_{s \mid n} \cap G_{s \mid n} \subset X$. It follows that $y=f(x) \in Y$, and that completes the proof.

\section{BI-ČECH-ANALYTIC SPACES}

A completely regular Hausdorff space is said to be an absolute Borel space if it is a Borel set in every compactification. To our knowledge, it is not known if a space that is a Borel set in some compactification is necessarily an absolute Borel space. Talagrand [8] has given an example of a $\mathscr{K}_{\sigma \delta}$ subset of a compact Hausdorff space which is not a $\mathscr{K}$-Borel set in some compactification (the $\mathscr{K}$ Borel sets of a space are the smallest collection containing the compact subsets and closed to countable unions and countable intersections). Here we describe a $\sigma$-algebra of Čech-analytic subsets of a compact Hausdorff space, containing all Borel sets and having an additional absolute embedding property (see Theorem 3.3). 
Definition 3.1. A completely regular Hausdorff space $X$ is bi- $\check{C} e c h$-analytic if both $X$ and $\beta X \backslash X$ are Čech-analytic spaces, where $\beta X$ is the Stone-Čech compactification of $X$.

Theorem 3.2. For a completely regular Hausdorff space $X$ the following are equivalent.

(a) $X^{*} \backslash X$ is a Čech-analytic space for some compactification $X^{*}$ of $X$.

(b) $X^{*} \backslash X$ is a Čech-analytic space for every compactification $X^{*}$ of $X$.

Proof. It suffices to show that (a) $\rightarrow($ b) . Suppose (a) holds, and let $f: \beta X \rightarrow$ $X^{*}$ be the continuous extension to $\beta X$ of the identity map on $X$. Since $f(\beta X \backslash X) \subset X^{*} \backslash X\left[1\right.$, p. 239], it follows that $\beta X \backslash X=f^{-1}\left(X^{*} \backslash X\right)$ and so is a Souslin- $(\mathscr{F} \wedge \mathscr{G})$ set in $\beta X$, since $X^{*} \backslash X$ is a Souslin- $(\mathscr{F} \wedge \mathscr{G})$ set in $X^{*}$. It follows that $\beta X \backslash X$ is a Čech-analytic space.

Now suppose $\beta X \backslash X$ is Čech-analytic, and let $X^{*}$ be any compactification of $X$. Let $f: \beta X \rightarrow X^{*}$ be the continuous extension of the identity map on $X$. Since $f^{-1}\left(X^{*} \backslash X\right)=\beta X \backslash X$, the restriction of $f$ to $\beta X \backslash X$ is a perfect map, and it follows that $f(\beta X \backslash X)=X^{*} \backslash X$ is Čech-analytic by Theorem 2.2.

The following theorem is an immediate consequence of Theorem 3.2 and its proof.

Theorem 3.3. For a completely regular Hausdorff space $X$ the following are equivalent.

(a) $X$ is bi-Čech-analytic.

(b) $X$ and $X^{*} \backslash X$ are Cech-analytic spaces for some compactification $X^{*}$ of $X$.

(c) $X$ and $X^{*} \backslash X$ are Čech-analytic spaces for some compactification $X^{*}$ of $X$.

Furthermore, the perfect image of any bi-Čech-analytic space is again bi-Čechanalytic.

Note that the collection of all bi-Čech-analytic subsets of any absolute Borel space $X$ forms a $\sigma$-algebra containing the Borel subsets of $X$. For a separable complete metric space $X$, the bi-Čech-analytic subsets coincide with the Borel sets by the classical Lusin first separation theorem. For nonseparable complete metric spaces the bi-Čech-analytic subsets coincide with the smallest $\sigma$-algebra containing the Borel sets and closed also to $\sigma$-discrete unions (see [7, Theorem 6.28] for some recent results of this type). This raises the question of whether the bi-Čech-analytic subsets of a general compact Hausdorff space can be characterized as the smallest $\sigma$-algebra containing the Borel sets and closed to some topological union.

\section{ACKNOWLEDGMENT}

We wish to thank Professor Dennis Burke for some valuable discussions on this problem which helped us to isolate a certain property of compact sets described in Lemma 2.1. 


\section{REFERENCES}

1. R. Engelking, General topology, PWN-Polish Scientific Publishers, Warszawa, and Academic Press, New York, 1977.

2. D. Fremlin, Čech-analytic space, unpublished note, 1980.

3. _ Perfect maps from Čech-analytic spaces, unpublished note, 1983.

4. Z. Frolík, Čech-analytic space, Comment. Math. Carolin. 25 (1984), 368-370.

5. __ Refinement of perfect maps onto metric spaces and an application to Čech-analytic spaces, Topology Appl. 33 (1989), 77-84.

6. R. W. Hansell, On characterizing non-separable analytic and extended Borel sets as types of continuous images, Proc. London Math. Soc. 28 (1974), 683-699.

7. __ Descriptive topology, Recent Progress in General Topology, Elsevier, New York and Oxford, 1992.

8. M. Talagrand, Choquet simplexes whose set of extreme points is $K$-analytic, Ann. Inst. Fourier (Grenoble) 35 (1985), 195-206.

Mathematics Department, University of Connecticut, Storrs, Connecticut 06269

E-mail address: hansellQuconnvm.uconn.edu

E-mail address: shiho@uconnvm.uconn.edu 